\title{
Evaluation of Callosal Motor Fiber Location in the Human Brain by Diffusion Tensor Tractography Combined with Functional MRI
}

\author{
Dong-Hoon Lee, $\mathrm{MS}^{1)}$, Young-Keun Woo, PhD, $\mathrm{PT}^{2)}$, Cheol-Pyo Hong, $\mathrm{PhD}^{1)^{*}}$ \\ 1) Department of Radiological Science, College of Health Science, Yonsei University: 234 Maeji, Heungup, \\ Wonju, Gangwondo 220-710, Republic of Korea. TEL: +82 33-760-2955, FAX: +82 33-760-2815 \\ 2) Department of Physical Therapy, College of Alternative Medicine, Jeonju University
}

\begin{abstract}
Purpose] The corpus callosum is the largest fiber bundle in the human brain that connects the two cerebral hemispheres. Callosal motor fiber is known to primarily cross through the anterior midbody of the corpus callosum. However, some reports have shown that callosal motor fibers cross through the posterior body and isthmus. In this study, we evaluated the relative anatomical location of callosal motor fibers related to foot fibers in the corpus callosum and collected quantitative data. [Methods] Fourteen healthy subjects participated in this study. A dataset was obtained utilizing a $1.5 \mathrm{~T}$ magnetic resonance imaging (MRI) scanner. For fiber tracking, regions of interest were drawn in the functional MRI activation area and corpus callosum. After the callosal motor fiber tract was reconstructed, we measured its relative anatomical locations in the corpus callosum, and calculated the fractional anisotropy (FA), and apparent diffusion coefficient (ADC) values. [Results] The mean \pm standard deviation of the distance ratio was $29.08 \pm 3.42 \%$. The values of FA and ADC were $0.54 \pm 0.02$ and $8.09 \times 10^{-4} \pm 0.22 \times 10^{-4}$, respectively. [Conclusion] We believe that our results present good preliminary data for the determination of the anatomical location of callosal motor fibers in the corpus callosum.

Key words: Callosal motor fiber, Corpus callosum, Diffusion tensor tractography
\end{abstract}

(This article was submitted Oct. 11, 2012, and was accepted Nov. 20, 2012)

\section{INTRODUCTION}

The corpus callosum (CC) is a crucial structure in the human brain. Being the largest fiber bundle, it enables interconnection between the two cerebral hemispheres ${ }^{1-8)}$. Previous studies have used magnetic resonance imaging (MRI) to determine several anatomical and morphological attributes of the $\mathrm{CC}$, such as volume, gender difference, and handedness for the evaluation of changes between health and neurological disorders $1,4,6,9-12)$. The anatomical location and features of CC-related motor fibers, known as callosal motor fibers (CMFs), have been studied by many neurological researchers to assess and obtain more accurate characteristic information about the $\left.\mathrm{CC}^{2}, 7,13-15\right)$. Through prior assessment of CMFs in the $\mathrm{CC}$, it is known that CMFs primarily cross through the anterior midbody of the CC, as shown in rhesus monkeys by Pandya et al. and Schmahmann et al. ${ }^{11,14)}$. However, Meyer et al. found that CMFs cross through the posterior body and isthmus of the CC utilizing transcranial magnetic stimulation (TMS) measurements, and Hofer et al., Zarei et al., and Wahl et al. made the same observations using diffusion tensor imaging $(\mathrm{DTI})^{2,7,8,13)}$. In essence, prior studies aiming to obtain accurate anatomical and functional features of the human brain have reported controversial results. Moreover, detailed information for CMFs in the CC is still limited and it is im-

*To whom correspondence should be addressed.

E-mail: hcpapa@naver.com portant to confirm the somatotopical organization of CMFs in the CC.

Many prior studies of the CC have used the DTI technique $\left.^{2,3}, 6,7,16-19\right)$. DTI provides a noninvasive method for visualizing white matter structures in the brain. Diffusion tensor tractography (DTT), based on DTI data, enables 3D neural fiber tract visualization, and has been used to identify major white matter pathways, such as the corticobulbar, corticospinal, and associated fiber tracts $\left.{ }^{8,} 16,18,19\right)$. Moreover, combining DTT with functional magnetic resonance imaging (fMRI) provides more accurate information for in vivo analysis of the brain ${ }^{7,20-22)}$. This method enables identification of the locations of cortical activation-related motor outcomes and helps set up a region of interest (ROI) by avoiding user-dependent bias.

In this study, we investigated CMFs related to foot movement in the human CC and measured the relative anatomical location using DTT in combination with fMRI. We also calculated the fractional anisotropy (FA) and apparent diffusion coefficient (ADC) values to provide quantitative information on normal ranges of CMF in the human $\mathrm{CC}$.

\section{SUBJECTS AND METHODS}

Fourteen healthy right-handed subjects ( 9 males and 5 females; mean age, 38.5 years) participated in this study. They had no previous history of neurological or physical disorders. All subjects provided their signed informed consent prior to their participation in the study. The study pro- 
tocol was approved by our local institutional review board.

DTI and fMRI datasets were obtained using a 1.5-T MRI scanner (Gyroscan Intera; Philips Healthcare, Best, Netherlands) with a six-channel phased-array sensitivity-encoding (SENSE) head coil and a reduction factor (r-factor) of 2 . Head motion was minimized with restraining foam pads provided by the manufacture ${ }^{23}$ )

The fMRI was performed using a blood oxygenation level-dependent (BOLD) sensitive echo planar imaging (EPI) sequence using the following parameters: time of repetition $(\mathrm{TR}) / \mathrm{time}$ of echo $(\mathrm{TE})=2000 / 60 \mathrm{~ms}$, field of view $(\mathrm{FOV})=$ $210 \mathrm{~mm}$, matrix size $=64 \times 64$, and slice thickness $=5 \mathrm{~mm}$. Subjects performed a simple visually guided motor task using instructions projected on a screen mounted on the rear end of the head coil ${ }^{7}$ ) The motor task performed was a foot movement involving the grasp and release of toes at a frequency of $1 \mathrm{~Hz}$, following a block paradigm. The activation and rest tasks were repeated three times and each task lasted $21 \mathrm{~s}$.

For DTI, imaging pulse sequences were based on two diffusion-sensitizing gradients from the single-shot spin echo EPI pulse sequence. The imaging parameters were as follows: $\mathrm{TR} / \mathrm{TE}=10726 / 75 \mathrm{~ms}, \mathrm{FOV}=221 \mathrm{~mm}$, matrix size $=128 \times 128$, and slice thickness $=2.3 \mathrm{~mm}$, with $63-67$ contiguous transverse slices with no slice gaps to cover the entire brain. A diffusion-weighting gradient was applied along 32 distinct directions with a b value of $1000 \mathrm{~s} / \mathrm{mm}^{2}$.

The fMRI data were processed using SPM2 (The Wellcome Department of Cognitive Neurology, London, UK) with familywise error (FWE) and $p<0.05$. Before statistical analysis, an image registration process was performed for motion correction. Since the statistically analyzed fMRI activation results used a ROI setting for fiber tracking, fMRI images were registered based on non-diffusion weighted images $\left(b=0 \mathrm{~s} / \mathrm{mm}^{2}\right)$. An 8-mm full-width at half-maximum (FWHM) Gaussian kernel was used to smooth the image during the fMRI analysis process.

The DTI data were analyzed using DtiStudio 3.0.2 software (Johns Hopkins University, Baltimore, Maryland, USA) that uses fiber assignment in a continuous tracking (FACT) algorithm ${ }^{24)}$. Before the diffusion parameter calculation and fiber tracking, the DTI datasets were subjected to an image distortion correction process to compensate for the effects of eddy current and head motion artifacts. All DTI images were registered to $b=0$ images using a twelve-parameter affine registration from the FMRIB software library (FSL, http://www.fmrib.ox.ac.uk/fsl) ${ }^{6-8)}$. The termination criteria for fiber tracking were as follows: FA $<0.2$ and angle change $>70^{\circ}$. Fiber tracking was started from a ROI that covered the foot representations, as defined by fMRI activation results related to foot movement and a second ROI was placed within the area of the $\mathrm{CC}$ in the color-coded FA map. After a CMF was reconstructed, we measured the relative fiber tract location by using the overlaid fiber tracts on each subject's $b=0$ image. The relative anatomical location of a CMF was measured as follows. (1) The entire CC distance, which is the distance between the most anterior point and posterior point of each subject, was measured in the sagittal plane. (2) The distance between the innermost point of the fiber and the most posterior point of $\mathrm{CC}$ was also measured. (3) The relative anatomical location

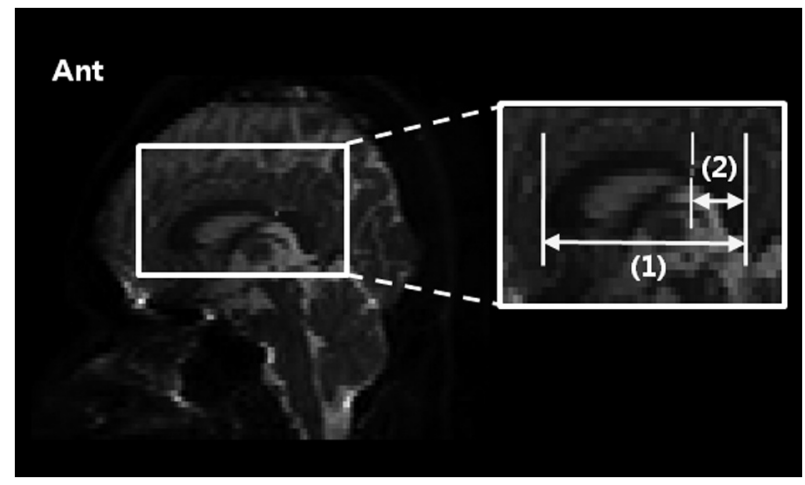

Fig. 1. Measurement of the relative anatomical location of callosal motor fibers (CMFs) related to foot movement in the $\mathrm{CC}$ The location was measured using the distance ratio between the entire distance of the CC in the sagittal plane (1) and distance from the innermost point of the CMF tract to the most posterior point of the CC (2).

was presented as the ratio of (1) and (2). These procedures are shown in Fig. 1. For quantification analysis, the FA and ADC values were calculated on the basis of the extracted foot movement CMFs from all voxels within the entire extracted CMF pathway of each subject.

\section{RESULTS}

The mean \pm SD of the measured relative anatomical location ratio of the CMF-related foot movement was 29.08 $\pm 3.42 \%$. This result indicats that foot CMFs are generally located in the posterior part of the $\mathrm{CC}$, occupying approximately one-third of its portion. In a previous study, Jancke et al. showed that the distance ratio of the isthmus was generally located in $20-33.3 \%$ of the posterior portion of the $\mathrm{CC}$, based on dividing the length of the structure into three equal portions, namely, the anterior third, middle third, and posterior third, including the isthmus and splenium ${ }^{9}$. Based on this result, we attempted to identify a more detailed relative anatomical location within the isthmus, which is located in the posterior third portion of the CC. Our individual result distribution was $64 \pm 8.93 \%$ (mean $\pm \mathrm{SD}$ ) based on the total length of the isthmus. This result shows that each individual CMF-related foot movement was strictly located in the posterior part of the isthmus. The mean $\pm \mathrm{SD}$ values for FA and ADC were $0.54 \pm 0.02$ and $8.09 \times 10^{-4} \pm 0.22 \times$ $10^{-4}$, respectively.

\section{DISCUSSION}

Many studies have investigated the anatomical and functional characteristic of the CC in humans $\left.{ }^{2}, 7,9,10,13,16,18,25\right)$. These studies investigated the thickness, volume, and area information of the $\mathrm{CC}$ to provide accurate anatomical information $\left.{ }^{9}, 10,25\right)$. Moreover, studies have compared the distribution of the CC in both healthy and diseased individuals in order to provide information regarding anatomical changes in diseased individuals ${ }^{1,11,26)}$ Although many studies have been performed so far, the anatomical location of CMFs in the $\mathrm{CC}$ is still a controversial topic. Previous studies have indicated that CMFs are located in the anterior portion of 
the $\mathrm{CC}^{14,15)}$. However, some previous studies have also reported conflicting results, indicating that CMFs in humans run in the posterior part of the $\mathrm{CC}^{2,7,8,13)}$. Therefore, it is important to elucidate the accurate location of CMFs in the $\mathrm{CC}$ to aid in the management of patients and to establish accurate information related to motor movement.

In this study, we evaluated the relative location and quantitative details of CMFs related to foot movement in the CC using a combined DTT and fMRI technique. The DTT/fMRI combination technique improved fiber tracking accuracy since the fMRI activation results used a ROI for fiber tracking. This combination approach was one of the methods utilized to reduce the erroneous factor-induced user-dependent ROI setting. Our findings confirmed those of prior research, suggesting that foot CMFs are located in the posterior part of the $\mathrm{CC}$ and within the isthmus. Using this result, we investigated the precise location of foot CMFs in the isthmus and obtained results showing a more specific location in the posterior part of the isthmus.

Although we used the combined DTT and fMRI technique to overcome erroneous results, this study still had a few limitations. The first was the limitation of the fiber-tracking algorithm utilized in this study. We used the FACT algorithm based on neural fiber line propagation, which depends on the principal axis of the eigenvector associated with the largest eigenvalue of the diffusion tensor. Therefore, this method will generate invalid results in the fiber-crossing regions. In a future study, we will use other tracking algorithms, such as probabilistic approaches, to circumvent this issue. Probabilistic algorithms allow the estimation of tensor direction, which is not considered a dominant direction and head to be multidirectional ${ }^{27}$. Additional limitations of this study were the small number of subjects and the failure to include other areas of motor movement, such as the hand or lip. Our future study will incorporate a larger number of subjects and investigate other somatotopic areas in order to create a more accurate and complementary study of CMFs in the CC.

In conclusion, we evaluated and measured the foot CMFs in the CC to identify their location and obtain quantitative information. Our results closely match those of previous research indicating that CMFs are located in the posterior third of the CC and within the isthmus. We believe that our results provide good preliminary data enabling the identification of the detailed anatomical location of CMFs in the $\mathrm{CC}$, and will provide valuable information to neuroscientists and researchers who study patients with foot movement diseases using CC management. Our results will also allow us to elucidate a more accurate neural pathway for foot movements. These results provide useful information in terms of rehabilitation, clinical physical therapy planning, and follow-up studies for a CMF neurological recovery mechanism in patients with $\mathrm{CC}$ damage that affects foot movement.

\section{REFERENCES}

1) Bellani M, Yeh PH, Tansella M, et al.: DTI studies of corpus callosum in bipolar disorder. Biochem Soc Trans, 2009, 37: 1096-1098. [Medline] [CrossRef]
2) Hofer S, Frahm J: Topography of the human corpus callosum revisitedcomprehensive fiber tractography using diffusion tensor magnetic resonance imaging. Neuroimage, 2006, 32: 989-994. [Medline] [CrossRef]

3) Huang H, Zhang J, Jiang H, et al.: DTI tractography based parcellation of white matter: application to the mid-sagittal morphology of corpus callosum. Neuroimage, 2005, 26: 195-205. [Medline] [CrossRef]

4) Lee BY, Sohn JH, Choi MH, et al.: A volumetric study of the corpus callosum in 20s and 40s Korean people. Brain Struct Funct, 2009, 213: 463-467. [Medline] [CrossRef]

5) Mooshagian E: Anatomy of the corpus callosum reveals its function. J Neurosci, 2008, 28: 1535-1536. [Medline] [CrossRef]

6) Park JS, Yoon U, Kwak KC, et al.: The relationships between extent and microstructural properties of the midsagittal corpus callosum in human brain. Neuroimage, 2011, 56: 174-184. [Medline] [CrossRef]

7) Wahl M, Lauterbach-Soon B, Hattingen E, et al.: Human motor corpus callosum: topography, somatotopy, and link between microstructure and function. J Neurosci, 2007, 27: 12132-12138. [Medline] [CrossRef]

8) Zarei M, Johansen-Berg H, Smith S, et al.: Functional anatomy of interhemispheric cortical connections in the human brain. J Anat, 2006, 209: 311-320. [Medline] [CrossRef]

9) Jäncke L, Staiger JF, Schlaug G, et al.: The relationship between corpus callosum size and forebrain volume. Cereb Cortex, 1997, 7: 48-56. [Medline] [CrossRef]

10) Luders E, Narr KL, Zaidel E, et al.: Gender effects on callosal thickness in scaled and unscaled space. Neuroreport, 2006, 17: 1103-1106. [Medline] [CrossRef]

11) Rutgers DR, Fillard P, Paradot G, et al.: Diffusion tensor imaging characteristics of the corpus callosum in mild, moderate, and severe traumatic brain injury. AJNR Am J Neuroradiol, 2008, 29: 1730-1735. [Medline] [CrossRef]

12) Witelson SF: Hand and sex differences in the isthmus and genu of the human corpus callosum. A postmortem morphological study. Brain, 1989, 112: 799-835. [Medline] [CrossRef]

13) Meyer BU, Roricht S, Woiciechowsky C: Topography of fibers in the human corpus callosum mediating interhemispheric inhibition between the motor cortices. Ann Neurol, 1998, 43: 360-369. [Medline] [CrossRef]

14) Pandya DN: The topography of commissural fibers. In: Two hemispheresone brain: functions of the corpus callosum. New York: Liss, 1986.

15) Schmahmann JD: Corpus callosum. In: Fiber pathways of the brain. New York: Oxford UP, 2006.

16) Abe O, Masutani $Y$, Aoki S, et al.: Topography of the human corpus callosum using diffusion tensor tractography. J Comput Assist Tomogr, 2004, 28: 533-539. [Medline] [CrossRef]

17) Hagmann P, Thiran JP, Jonasson L, et al.: DTI mapping of human brain connectivity: statistical fibre tracking and virtual dissection. Neuroimage, 2003, 19: 545-554. [Medline] [CrossRef]

18) Hasan KM, Kamali A, Iftikhar A, et al.: Diffusion tensor tractography quantification of the human corpus callosum fiber pathways across the lifespan. Brain Res, 2009, 1249: 91-100. [Medline] [CrossRef]

19) Jarbo K, Verstynen T, Schneider W: In vivo quantification of global connectivity in the human corpus callosum. Neuroimage, 2012, 59: 19881996. [Medline] [CrossRef]

20) Conturo TE, Lori NF, Cull TS, et al.: Tracking neuronal fiber pathways in the living human brain. Proc Natl Acad Sci USA, 1999, 96: 10422-10427. [Medline] [CrossRef]

21) Dougherty RF, Ben-Shachar M, Bammer R, et al.: Functional organization of human occipital-callosal fiber tracts. Proc Natl Acad Sci USA, 2005, 102: 7350-7355. [Medline] [CrossRef]

22) Kim DS, Kim M: Combining functional and diffusion tensor MRI. Ann N Y Acad Sci, 2005, 1064: 1-15. [Medline] [CrossRef]

23) Kim DJ, Park HJ, Kang KW, et al.: How does distortion correction correlate with anisotropic indices? A diffusion tensor imaging study. Magn Reson Imaging, 2006, 24: 1369-1376. [Medline] [CrossRef]

24) Jiang H, van Zijl PC, Kim J, et al.: DtiStudio: resource program for diffusion tensor computation and fiber bundle tracking. Comput Methods Programs Biomed, 2006, 81: 106-116. [Medline] [CrossRef]

25) Johnson SC, Farnworth T, Pinkston JB, et al.: Corpus callosum surface area across the human adult life span: effect of age and gender. Brain Res Bull, 1994, 35: 373-377. [Medline] [CrossRef]

26) Yeatman JD, Ben-Shachar M, Bammer R, et al.: Using diffusion tensor imaging and fiber tracking to characterize diffuse perinatal white matter injury: a case report. J Child Neurol, 2009, 24: 795-800. [Medline] [CrossRef]

27) Behrens TE, Berg HJ, Jbabdi S, et al.: Probabilistic diffusion tractography with multiple fibre orientations: what can we gain? Neuroimage, 2007, 34: 144-155. [Medline] [CrossRef] 